CERN-EP/2002-070

17 September 2002

\title{
Atomic and Nuclear Physics with Stored Particles in Ion Traps
}

\author{
H.-Jürgen Kluge ${ }^{122)}$, Klaus Blaum ${ }^{1)}$, Frank Herfurth ${ }^{3)}$ and Wolfgang Quint ${ }^{1)}$
}

\begin{abstract}
Trapping and cooling techniques play an increasingly important role in many areas of science. This review concentrates on recent applications of ion traps installed at accelerator facilities to atomic and nuclear physics such as mass spectrometry of radioactive isotopes, weak interaction studies, symmetry tests, determination of fundamental constants, laser spectroscopy, and spectroscopy of highly-charged ions. In addition, ion traps are proven to be extremely efficient devices for (radioactive) ion beam manipulation as, for example, retardation, accumulation, cooling, beam cleaning, charge-breeding, and bunching.
\end{abstract}

(Submitted to: Physica Scripta, 2002)

${ }^{1}$ Gesellschaft für Schwerionenforschung (GSI), D-64291 Darmstadt, Germany

${ }^{2}$ Universität Heidelberg, D-69120 Heidelberg, Germany

${ }^{3}$ CERN, Division EP/ISOLDE, CH-1211 Geneva, Switzerland 


\section{Introduction}

Since quite a long time, starting in the 50ies of last century, ion traps are widely applied to atomic physics, to the determination of fundamental constants, metrology, mass spectrometry, trace analysis and chemistry. However, only quite recently, ion traps are also used in nuclear research and are installed at accelerator facilities. The latter developments were prompted by two achievements:

- The measurements by the Penning trap mass spectrometer ISOLTRAP [1,2] at the on-line isotope separator ISOLDE/CERN [3] demonstrated that a mass resolving power of $10^{7}$ and a relative mass accuracy of $10^{-8}$ could be achieved [4] by use of an ion trap coupled to a radioactive ion beam (RIB) facility.

- The gas-filled segmented linear Paul trap was shown by groups from Argonne, CERN, GSI, Jyväskylä, Montreal, Munich and Orsay [2,5-7] to be an efficient tool for retardation, accumulation, cooling, and bunching of ion beams delivered by RIB facilities.

Today, quite a number of ion traps are in operation, under construction, or planned at accelerators (Table1). A general overview can be obtained by the proceedings of the following conferences: "Trapped Charged Particles and Fundamental Physics", Asilomar, 1998 [8], "Atomic Physics at Accelerators: Mass Spectrometry (APAC 2000)", Cargèse, 2000 [9], "14 $4^{\text {th }}$ International Conference on Electromagnetic Isotope Separation and Techniques Related to their Application (EMIS-14)", Victoria, 2002 [10], and "Trapped Charged Particles and Fundamental Physics", Wildbad Kreuth, 2002 [11]. In the following, the principle of Penning and Paul traps is briefly described and the physics motivation for the different ion trap facilities at accelerators, their status as well as some recent results are presented.

\section{Principle of Ion Traps}

\subsection{Penning Traps}

The storage of charged particles in a Penning trap is achieved by the superposition of a homogeneous magnetic field serving for radial confinement and an axially symmetric electrostatic quadrupole field, which prevents the ions from escaping along the magnetic field lines [12,13]. In general, this configuration is achieved by three hyperboloidal electrodes, two end electrodes and a ring electrode, or by cylindrical electrodes with appropriate potentials applied. Both devices are schematically shown in Fig. 1.

Due to the electric quadrupole field the motion of the ion with mass $m$ and charge state $q$ is not only a pure cyclotron motion with frequency $\omega_{c}=(q / m) B$ in a magnetic field $B$, but a superposition of three harmonic eigenmotions, as shown in Fig. 2: a slow drift around the trap axis, called magnetron motion with frequency $\omega_{-}$, a modified (or reduced) cyclotron motion with frequency $\omega_{+}$and a $z$ (or axial) oscillation with frequency $\omega_{z}$. For an ideal electric quadrupole field with a potential difference of $U_{o}$ between the ring and the end electrodes in a uniform magnetic field $B$ these eigenfrequencies are: 
Table 1: Ion traps at accelerator facilities in operation (op.), under construction or test (uc.), or planned ( $\mathrm{pl}$.) for atomic and nuclear physics. In the upper part of the table, the ion traps for weak interaction studies are compiled and the main isotopes under investigation are indicated. In the middle, the Penning trap facilities at the Antiproton Decelerator AD at CERN are listed aiming at a CPT test by comparing the $1 \mathrm{~s}-2 \mathrm{~s}$ transition in hydrogen and antihydrogen. In the lower part the name of the ion trap facility is given in the first column. The principal goals are indicated by at $=$ atomic spectroscopy, $c b=$ charge breeding, $c h=$ chemistry, Is = laser spectroscopy, ms = mass spectrometry, ns = nuclear spectroscopy. The trapping device is indicated by: $\mathrm{GC}=$ gas cell, RFQ = gas-filled segmented radio frequency quadrupole trap, EBIS = electron beam ion source.

\begin{tabular}{|c|c|c|c|c|c|}
\hline Isotope/Name & Goal & Institute & Kind of Trap & Status & Ref. \\
\hline $\begin{array}{l}{ }^{6} \mathrm{He} \\
{ }^{14} \mathrm{O} \\
{ }^{35} \mathrm{Ar} \\
{ }^{77} \mathrm{Kr}\end{array}$ & $\begin{array}{l}\beta-\nu \text { correlation } \\
\beta-\nu \text { correlation } \\
\beta-\nu \text { correlation } \\
\text { bound- } \beta \text { decay }\end{array}$ & $\begin{array}{l}\text { GANIL/Caen } \\
\text { Argonne Nat. Lab. } \\
\text { ISOLDE/CERN, Leuven } \\
\text { LBL }\end{array}$ & $\begin{array}{l}\text { Paul trap } \\
\text { Paul trap } \\
\text { Penning trap } \\
\text { Penning Trap/EBIT }\end{array}$ & $\begin{array}{l}\text { uc. } \\
\text { uc. } \\
\text { uc. } \\
\text { uc. }\end{array}$ & $\begin{array}{l}{[16]} \\
{[17]} \\
{[18]} \\
{[19]}\end{array}$ \\
\hline $\begin{array}{l}\text { ATHENA } \\
\text { ATRAP }\end{array}$ & $\begin{array}{l}\text { CPT test } \\
\text { CPT test }\end{array}$ & $\begin{array}{l}\text { AD/CERN } \\
\text { AD/CERN }\end{array}$ & $\begin{array}{l}\text { Penning trap } \\
\text { Penning trap }\end{array}$ & $\begin{array}{l}\text { op. } \\
\text { op. }\end{array}$ & $\begin{array}{l}20] \\
{[21]}\end{array}$ \\
\hline $\begin{array}{l}\text { CPT } \\
\text { HITRAP } \\
\text { ISOLTRAP } \\
\text { JYFL-TRAP } \\
\text { LEBIT } \\
\text { MAFF-TRAP } \\
\text { REX-ISOLDE } \\
\text { RIKEN-TRAP } \\
\text { SHIPTRAP } \\
\text { SMILETRAP } \\
\text { TITAN } \\
\end{array}$ & $\begin{array}{l}\text { Is, ms, ns } \\
\text { as, Is, ms } \\
\text { ms } \\
\text { ls, ms, ns } \\
\mathrm{ms}, \mathrm{ns} \\
\mathrm{ch}, \mathrm{ms}, \mathrm{ns}, \\
\mathrm{cb} \\
\mathrm{ns}, \mathrm{ls} \\
\mathrm{ms}, \mathrm{ls}, \mathrm{ch}, \mathrm{ns} \\
\mathrm{ms} \\
\mathrm{ms}, \mathrm{ls}, \mathrm{cb}, \mathrm{as}, \mathrm{ns}\end{array}$ & $\begin{array}{l}\text { Argonne/Chicago } \\
\text { GSI/Darmstadt; Mainz } \\
\text { ISOLDE/CERN } \\
\text { Jyväskylä } \\
\text { MSU/East Lansing } \\
\text { Munich } \\
\text { ISOLDE/CERN } \\
\text { RIKEN/Tokyo } \\
\text { GSI/Darmstadt } \\
\text { MSL/Stockholm } \\
\text { TRIUMF/Vancouver } \\
\end{array}$ & $\begin{array}{l}\text { GC/RFQ/Penning trap } \\
\text { Penning trap } \\
\text { RFQ/Penning trap } \\
\text { RFQ/Penning trap } \\
\text { GC/RFQ/Penning trap } \\
\text { RFQ/Penning trap } \\
\text { Penning trap/EBIS } \\
\text { GC/RFQ/combined trap } \\
\text { GC/RFQ/Penning trap } \\
\text { Penning trap } \\
\text { RFQ/EBIS/Penning trap }\end{array}$ & $\begin{array}{l}\text { op. } \\
\text { pl. } \\
\text { op. } \\
\text { op/uc. } \\
\text { pl. } \\
\text { pl. } \\
\text { uc. } \\
\text { op/uc. } \\
\text { uc. } \\
\text { op. } \\
\text { pl. }\end{array}$ & $\begin{array}{c}{[22]} \\
{[23]} \\
{[1,2]} \\
{[6,24]} \\
{[25]} \\
{[26]} \\
{[27]} \\
{[28]} \\
{[29]} \\
{[30]} \\
{[31]} \\
\end{array}$ \\
\hline
\end{tabular}
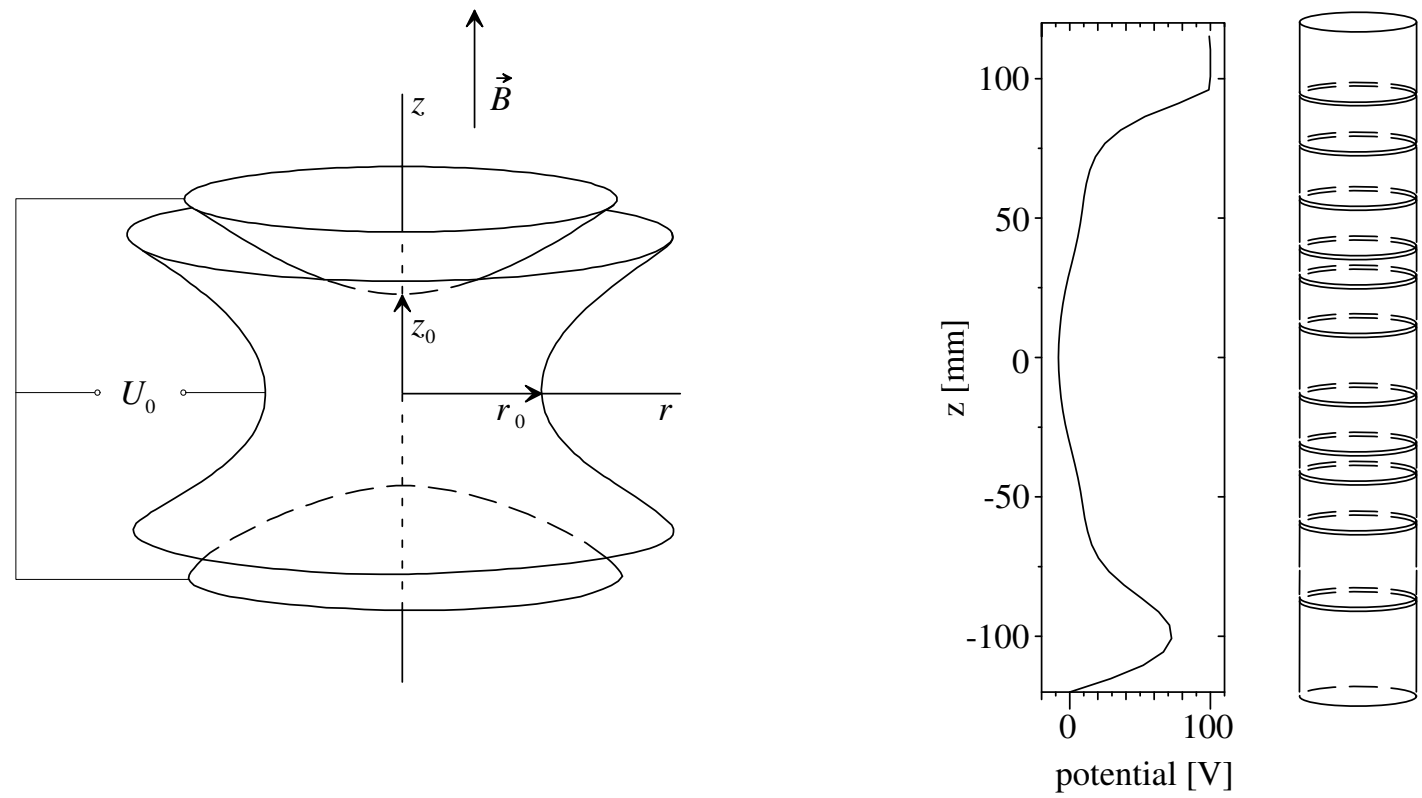

Figure 1: Basic configurations of a Penning trap, consisting of two end electrodes and a ring electrode with hyperboloidal shapes (left) or of cylindrical electrodes (right). For the case of the cylindrical Penning trap, the slope of the electric potential along the magnetic field axis is indicated. A harmonic potential is created around $z=0$ whereas the extended potential well is used for efficient capture of injected ions. 


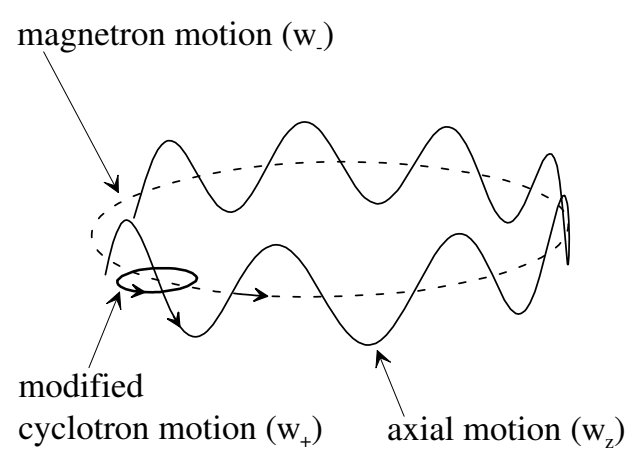

Figure 2: Eigenmotions (left) and energy levels (right) of a charged particle in an ideal Penning trap. The total energy is given by the sum of the energies of the three independent harmonic oscillations. The potential energy is set to zero in the center of the trap. Note that the magnetron motion has mainly potential energy: Exciting the magnetron motion results in decreasing the total energy. Applying a dipole electric field changes the quantum number in one of the ladders of the Landau levels. An RF quadrupole electric field results in coupling the Landau levels of two different ladders.

the z-oscillation with frequency

$$
\omega_{\mathbf{z}}=\sqrt{\frac{\mathbf{q u}_{\mathbf{o}}}{\mathbf{m d}^{2}}},
$$

the modified cyclotron oscillation

$$
\omega_{+}=\frac{\omega_{\mathrm{c}}}{2}+\sqrt{\frac{\omega_{\mathrm{c}}^{2}}{4}-\frac{\omega_{\mathrm{z}}^{2}}{2}}
$$

and the magnetron oscillation

$$
\omega_{-}=\frac{\omega_{c}}{2}-\sqrt{\frac{\omega_{c}^{2}}{4}-\frac{\omega_{z}^{2}}{2}}
$$

\subsubsection{Cooling of lons in a Penning Trap}

Cooling means to lower the quantum numbers $n$ of the particles oscillating in the trap potential as shown in the right part of Fig. 2. Quite a number of different cooling techniques have been developed during the past years. Here, only those cooling methods will be briefly discussed which are applied to charged particles stored in Penning traps installed at accelerators.

The most common cooling technique for radioactive ions is buffer-gas cooling in combination with radio-frequency (RF) excitation at the true cyclotron frequency $\omega_{c}$. The cyclotron as well as the axial oscillations are damped by buffer gas collisions. 
However, the magnetron orbit increases since the ion loses (potential) energy by collisions with the buffer gas atoms. This drift to the ring electrode is counteracted by excitation of the stored ion by a quadrupolar RF-field that couples the modified cyclotron and the magnetron motion [14]. In this way, all three quantum numbers $n_{+}$, $n_{z}$ and also $n_{m}$ (or $n_{-}$), are driven to smaller numbers (Fig. 2) and the temperature of the ion cloud finally reaches that of the buffer gas. This cooling and centering technique, which is also mass selective, is applied or planned for CPT, ISOLTRAP, JYFL-TRAP, LEBIT, MAFF-TRAP, REXTRAP, SHIPTRAP, and TITAN (see Table 1 and [10]) and is now also used in many Fourier Transform lon Cyclotron Resonance (FT-ICR) experiments in chemistry [15].

Resistive cooling is applied in the experiments with antiprotons at the Antiproton Deccelerator (AD) at CERN and in the g-2 set-up of the HITRAP project at Mainz (Table 1). The motional energy of the stored charged particles is damped by use of a circuit, which is tuned to the eigenfrequency of the ion motion, and dissipated in a resistor of the circuit via the image currents induced in the trap electrodes. Finally, the energy of the ions corresponds to the temperature at which the resistor is kept (generally $4 \mathrm{~K}$ ).

A brute force technique as applied in SMILETRAP (Table 1) is evaporative cooling. Here, the trapping potential is lowered so that the ions with highest kinetic energy escape from the trap. The ions remaining in the shallow potential well have low motional energy once the trapping voltage is returned to normal values.

Electron cooling by Coulomb interaction with simultaneously stored electrons was found to be an effective cooling mechanism for antiprotons [32]. The electrons are self-cooling by synchrotron radiation in the high magnetic field of the Penning trap [12].

\subsection{Paul Traps}

In the absence of a magnetic field, particles can also be confined in three dimensions by a device as the one shown in Fig. 1 when instead of the DC-potential $U_{0}$ an RFfield $U=U_{D C}+U_{R F} \cdot \sin \left(\omega_{R F} t\right)$ is applied between endcaps and ring electrode instead of a static potential $U_{0}$. Such a storing device is called Paul trap or radio frequency quadrupole (RFQ) trap [33]. The interaction of the trapped ion with the time-varying quadrupole potential is described by the Mathieu equations. When the stability conditions are fulfilled, the ion is confined and performs a fast micromotion with frequency $\omega_{R F}$ and a slow macromotion.

Confinement in two dimensions is obtained in the Paul mass filter which is also often called ion guide and which is used, for example, in rest gas analysis or analytical chemistry. Here, the RF-potential is applied to four parallel rods with a phase shift of $180^{\circ}$ between two neighbouring electrodes. By a proper choice of $U_{D C}, U_{R F}$, and $\omega_{R F}$, only ions with a specific charge-to-mass ratio are transmitted.

Ions can be confined in such a linear structure also in three dimensions if the four rods are longitudinally segmented and appropriate DC potentials are applied to the segments. Figure 3 shows the segmented Paul filter as used at ISOLTRAP at ISOLDE. 


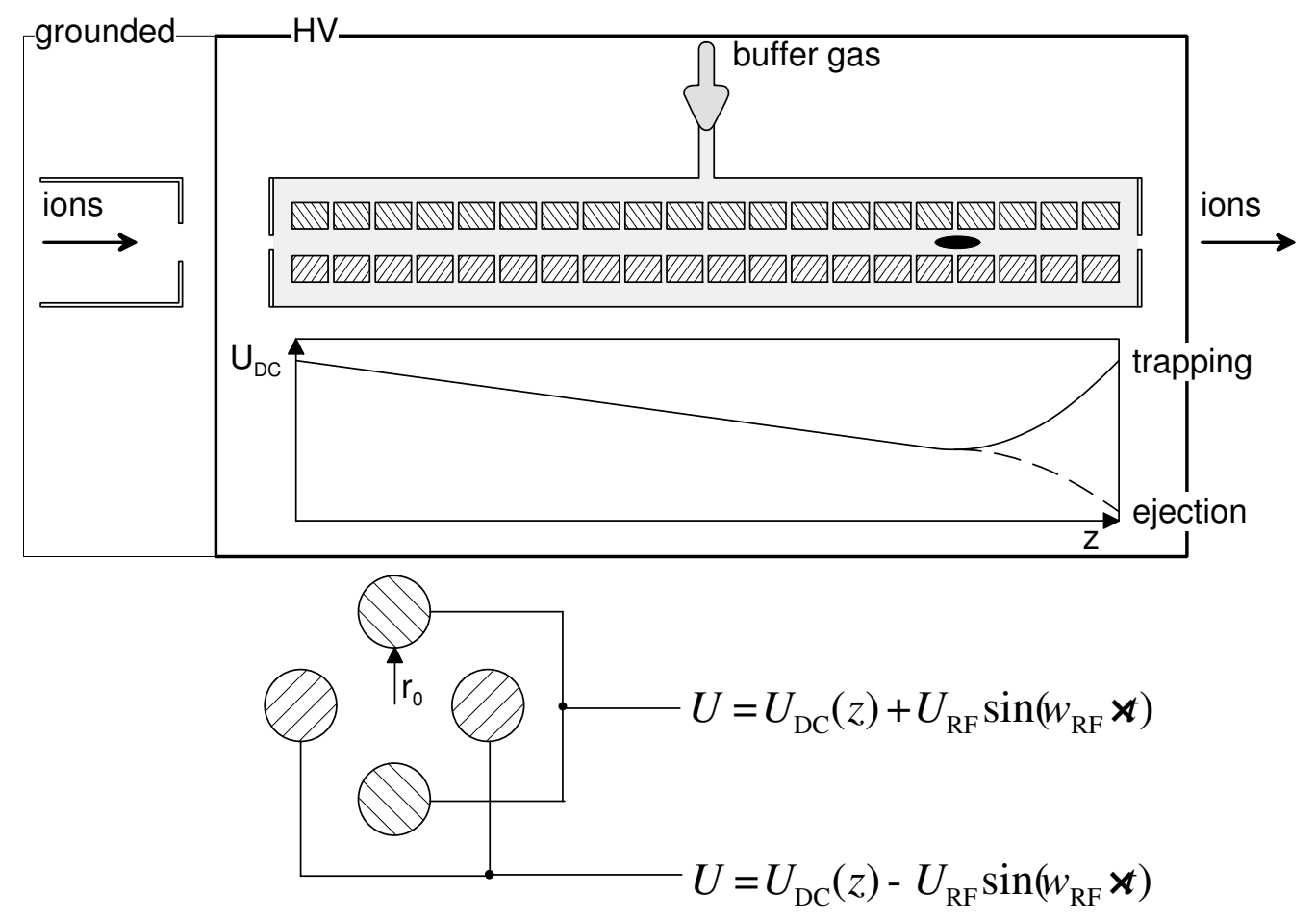

Figure 3: Segmented linear Paul trap as used at ISOLTRAP. The $60 \mathrm{keV}$ ions delivered by the on-line isotope separator are retarded by putting the hole set-up near to $60 \mathrm{keV}$ and filling the device with He buffer gas. Due to collisions with the buffer gas atoms, the ions are cooled within some milliseconds to nearly the temperature of the buffer gas.

\subsubsection{Cooling in Radio Frequency Quadrupole Traps}

Cooling in Paul traps or Paul filters is most easily achieved by collisions with buffer gas atoms. Cooling times of some milliseconds can be reached. Laser cooling, which is even faster and more efficient in terms of final temperature, is generally not applied to ion trap facilities at accelerators since every element requires a special cooling scheme.

\section{Beam Manipulation}

Ion traps are now quite often applied at accelerators for the manipulation of lowenergy radioactive beams. This includes retardation, accumulation, cooling, storing, beam cleaning, and bunching [2,5,6,22,27-29]. Ion trapping devices are also applied to charge breeding as in REX-ISOLDE, in order to achieve efficient post-acceleration of radioactive beams [27].

At ISOLDE [34] and at TRIUMF [35], RFQ coolers are under construction as general devices to improve the emittance of the delivered radioactive beams and in this way to increase the efficiency of quite a number of performed experiments. Furthermore, the application of ion traps is planned as storage device and source of decay particles without backing for weak interaction studies [16-19] and decay spectroscopy $[17,36]$.

In the TITAN facility an RFQ cooler will be used for cooling of radionuclides and bunched injection into an electron beam ion trap (EBIT) for charge breeding [31]. SHIPTRAP [29] and JYFL-Trap [24] are applying an RFQ cooler for an efficient 
injection into the Penning trap where further beam cleaning from isobaric contaminations will take place. Similar trap facilities are under construction at MSU [25] and are planned for the MAFF facility at the Munich reactor FRM II [26].

Penning traps are further in operation at the antiproton decelerator AD at CERN for accumulation and cooling of positrons and antiprotons with the final goal of antihydrogen production and a CPT test by laser spectroscopy in the 1s-2s transition $[20,21]$. In the planned HITRAP facility at GSI, highly-charged ions from the heavy ion synchrotron SIS, cooled and decelerated in the experimental storage ring ESR and further decelerated by a linear decelerator, are accumulated and cooled to $4 \mathrm{~K}$ in a Penning trap [23]. Experiments, such as the g-factor measurement described in Section 5.4, are planned for very heavy few-electron systems as for example $U^{91+}$.

A gas cell and a six-pole ion guide is presently being tested for stopping, accumulation, cooling, and transfer of radioactive beams from the RIKEN fragment separator to a RFQ trap. Later on, transfer to a combined trap (RFQ plus Penning trap) is planned for the determination of nuclear ground-state properties by laser spectroscopy [28].

Finally, a laser ion trap (LIST) is proposed and presently built up and tested at the University of Mainz [37]. The aim is to drastically improve the isobaric selectivity of laser ion sources at on-line isotope separators based on resonance ionization. As trapping and cooling in a linear RFQ structure is involved, LIST will deliver radioactive ion beams with selectable temporal structure from quasi-DC to pulses with microsecond length and with an emittance which is improved by an order of magnitude as compared to conventional laser ion sources at on-line isotope separators.

\section{Mass Spectrometry}

The main scientific goal pursued at most of the existing and planned Penning trap facilities at accelerators (Table 1 ) is mass spectrometry of radionuclides. The mass determination in a Penning trap is based on the determination of the cyclotron frequency

$$
\omega_{\mathrm{c}}=\frac{\mathbf{q}}{\mathbf{m}} \mathbf{B}
$$

of ions with mass $m$ and charge $q$ in a magnetic field of strength $B$.

In high-accuracy mass measurements the important relationships between the eigenfrequencies of the motion of the ion are

$$
\begin{aligned}
& \omega_{c}=\omega_{+}+\omega_{-}, \\
& \omega_{c}^{2}=\omega_{+}^{2}+\omega_{-}^{2}+\omega_{z}^{2} .
\end{aligned}
$$

The independent determination of the eigenfrequencies and the use of these equations allow a mass determination of the stored ions with only the magnetic field known. The eigenfrequencies can be determined by driving the corresponding motions by a dipole RF field. However, the most direct approach is the measurement 
of the sum frequency $\omega_{c}=\omega_{+}+\omega_{-}$which corresponds to a coupling of the modified cyclotron and magnetron oscillation. This can be achieved by an azimuthally quadrupolar RF field applied to a four-fold segmented ring electrode.

The magnetic field strength $B$ in the Penning trap is determined by measuring the cyclotron frequency of an ion with well-known mass. Recently it was shown that absolute mass measurements are possible by using carbon clusters for calibration [4].

\subsection{Detection of cyclotron resonance}

There are three main techniques in order to determine the mass via a measurement of the cyclotron frequency of the stored ions: broad-band or narrow-band observation of the image currents induced by the motion of the ions in the trap electrodes or the measurement of the time of flight (TOF) of the ions from the ion trap to an ion detector placed outside the magnetic field.

A broad-band observation of the masses of the confined ions is achievable by Fourier Transformation Ion Cyclotron Resonance (FT-ICR) spectroscopy [15] where the ion cloud is excited to large cyclotron orbits and the image signal is time-differentially recorded and finally Fourier transformed into the frequency domain.

A more sensitive detection scheme, allowing even the detection of a single ion, is to observe the image current via a tuned circuit of high quality factor $Q$. Generally, the axial oscillation is monitored. In order to detect changes in the cyclotron orbit, a coupling is required between the modified cyclotron and the axial motion, which are completely decoupled in the ideal trap. Coupling of motions can be achieved, for example, magnetically [12], electrostatically [38], or by side-band excitation [39]. A slight change in the cyclotron radius upon its excitation results in this way in a change of the axial motion.

Whereas the observation of the image currents is non-destructive, the TOF detection [40] of the cyclotron resonance is destructive. Here, the ions are ejected from the trap, drift along the magnetic field lines to a channel-plate detector, where they are detected, and are lost. This is generally not a drawback in the case of mass determination of radionuclides since the nuclei decay anyhow. Therefore, the TOF technique is applied up to now in all Penning trap mass spectrometers for radioactive nuclei which are operational or planned.

The TOF technique is based on the acceleration of a magnetic dipole in a magnetic field gradient. [40]. The ions are first excited to a magnetron orbit with small radius [1]. Here, the orbital frequency and, therefore, the orbital magnetic moment as well as the associated energy $E=\mu \cdot B$ is small. The magnetron motion is converted into (modified) cyclotron motion by a quadrupolar RF field with frequency $\omega_{\mathrm{RF}}=\omega_{\mathrm{c}}$ while the radius of the radial motion remains constant [13]. After full conversion to cyclotron motion, the orbital frequency, magnetic moment, and energy are much higher as compared to the initial magnetron motion leading to a longitudinal acceleration in the gradient of the magnetic field after ejection of the ions. Therefore, the TOF is shortened when the cyclotron resonance is hit. 


\subsection{Resolving power and accuracy}

The resolving power $R=v_{d} \Delta v_{c}(F W H M)$ of Penning trap mass spectrometers is ultimately Fourier limited. Either the period of time for coherent RF-excitation $T_{R F}$ or the observation time of the evolution of the cyclotron motion determine the line width by [1]

$$
\Delta v_{c}(F W H M) \approx 1 / T_{R F}
$$

In order to obtain a high resolving power, high cyclotron frequencies by strong magnetic fields or high charge states, and long excitation or observation times are desirable.

To give an example, a singly-charged ion with mass $m=100 u$ is considered which is confined in a Penning trap with a magnetic field strength of $B=6 \mathrm{~T}$. Then the cyclotron frequency is about $v_{C}=1 \mathrm{MHz}$. An excitation time of $T_{R F}=1 \mathrm{~s}$ results in a resolving power of typically $R=10^{6}$. Determining the center of the resonance to $10 \%$ of the full half width, a relative accuracy of $\delta v_{d} v_{c}=\delta \mathrm{m} / \mathrm{m}=10^{-7}$ is reached if the magnetic field strength is known.

For light ions, the achievable resolving power and accuracy are even higher. For the mass comparison of the proton and antiproton, for example, the above conditions yield $R=10^{8}$ and $\delta \mathrm{m} / \mathrm{m}=10^{-9}$. By prolonging the observation time, the mass of the proton and antiproton was found to be identical within $9 \cdot 10^{-11}$, which represents by far the best check of CPT-invariance in the case of baryons [41].

Presently, the achievable accuracy in mass measurements on stable particles or isotopes is around $\delta \mathrm{m} / \mathrm{m}=10^{-10}$. It is obtained either by a long observation period of time [41-44] or by usage of highly-charged ions [30]. This limit in accuracy is presently caused by fluctuations of the magnetic field which result in the above uncertainty when subsequently loading the ion under investigation and then the reference ion with well-known mass into the trap. Very recently, the MIT group succeeded in parking two different ion species in a magnetron orbit at the same time and to measure their cyclotron frequency quasi simultaneously [44]. This might result in an improvement of the achievable accuracy by about two orders of magnitude.

\subsection{Penning Trap Mass Spectrometer for keV radioactive beams}

Since the ions have to be injected, captured, and cooled for mass spectrometry in a Penning trap, it is advantageous to use radioactive ion beams of low energy and small emittance as provided by on-line isotope separators. Therefore, the first Penning trap for on-line mass spectrometry, ISOLTRAP [1,2], has been installed at the on-line isotope separator ISOLDE/CERN [3].

\subsubsection{ISOLTRAP}

The triple-trap mass spectrometer ISOLTRAP consists of three functional parts: a gas-filled segmented radio-frequency quadrupole (RFQ) ion trap for beam preparation and two Penning traps for cooling and for the mass measurement. A stable alkali reference ion source serves for calibration of the magnetic field. A very recent addition is a carbon cluster ion source $[4,45]$ where laser-produced fragments 
of $\mathrm{C}_{60}$ fullerenes can be used for absolute mass calibrations. In total, the masses of more than 200 short-lived nuclides have been measured with a relative uncertainty of $\delta m / m \approx 1 \cdot 10^{-7}$ (corresponding to $\delta m<10 \mathrm{keV}$ for $A<100$ ) [46-52] and close to $10^{-8}$ in some special cases $[52,4]$.

Figure 4 shows the ISOLTRAP set-up. The ISOLDE ion beam enters from the left with an energy of $60 \mathrm{keV}$. It is retarded, accumulated, and cooled in the RFQ structure put to a potential just below $60 \mathrm{kV}$ [2]. After a certain accumulation time (typically $10-20 \mathrm{~ms}$ ) the ions are accelerated as an ion bunch towards a drift tube which is switched down from about $57.5 \mathrm{kV}$ to ground potential when the ion bunch resides in it. The $2.5 \mathrm{keV}$-energy bunch is then transferred into the tandem Penning trap system and captured there in-flight. In the cooling Penning trap the ions are stored (up to 1s) and contaminant isobaric ions are removed. For this, a massselective buffer gas cooling technique is used [14]. The ions are then transferred to the second, precision Penning trap, where ground and isomeric states can be resolved and where the actual mass measurement is carried out by a determination of the cyclotron frequency via the time-of-flight technique. As an example, the cyclotron resonance of ${ }^{32} \mathrm{Ar}^{+}$is shown in the inset of Fig. 4 with a fit by the theoretical line shape [53].

With a resolving power of up to $10^{7}$ and an accuracy of maximum $10^{-8}$ quite a number of important mass measurements have been performed during recent years at ISOLTRAP. A few examples will be presented in the following.

Very recently, it has become possible to push the applicability of Penning trap mass measurements to radionuclides with half-lives well below one second. The nuclide with the shortest half-life measured until now is ${ }^{74} \mathrm{Rb}$ with a half-life $T_{1 / 2}$ of only $65 \mathrm{~ms}$ [52].

The mass of ${ }^{33} \mathrm{Ar}$ was determined by ISOLTRAP with an accuracy of $1 \cdot 10^{-7}$ [49]. The result was not consistent with the mass calculated by use of the isobaric multiplet mass equation (IMME). This discrepancy could have had severe consequences for the presently best exclusion test of scalar contributions to weak interaction as performed by Adelberger et al. [54] at ISOLDE by use of ${ }^{32} \mathrm{Ar}$. Therefore, these measurements prompted further investigations which now solved the problem in the case of ${ }^{33} \operatorname{Ar}[55]$.

In the meantime, also the mass of ${ }^{32} \mathrm{Ar}$ was measured by ISOLTRAP. Here, a precision of $\delta \mathrm{m} / \mathrm{m}<1 \cdot 10^{-7}$ (inset of Fig. 4) was reached despite a production rate of only about 100 ions/proton pulse [56]. The result differs from the IMME prediction used by Adelberger et al. [54] by nearly $3 \sigma$ and as a consequence a re-evaluation of the Adelberger experiment is under way.

The mass of ${ }^{34} \operatorname{Ar}\left(T_{1 / 2}=844 \mathrm{~ms}\right)$ was determined with a relative uncertainty of only $1.1 \cdot 10^{-8}$ [52]. This is the highest precision ever obtained in direct mass measurements of short-lived nuclides. With this result the $Q_{E C}$ value for the $\beta$ decay of ${ }^{34} \mathrm{Ar}$ was determined with an uncertainty well below $1 \mathrm{keV}$, sufficient to be used in a decisive test of Coulomb correction calculations for $\mathrm{ft}$-values. In the very same context, also the $Q$ value of ${ }^{74} \mathrm{Rb}$ is important. Here ISOLTRAP was able to measure the mass of ${ }^{74} \mathrm{Kr}$ with a precision of $3.2 \cdot 10^{-8}$, but due to limited statistics the mass of ${ }^{74} \mathrm{Rb}$ could only be obtained with an uncertainty of $2.6 \cdot 10^{-7}$ or $\delta m=18 \mathrm{keV}$. 


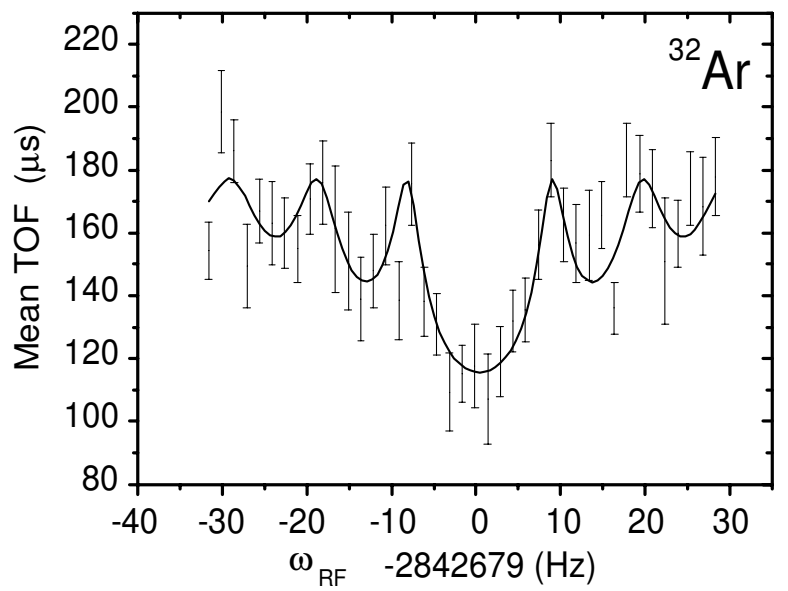

stable alkali ion reference source

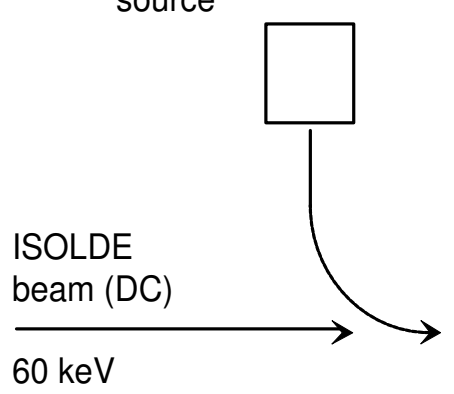

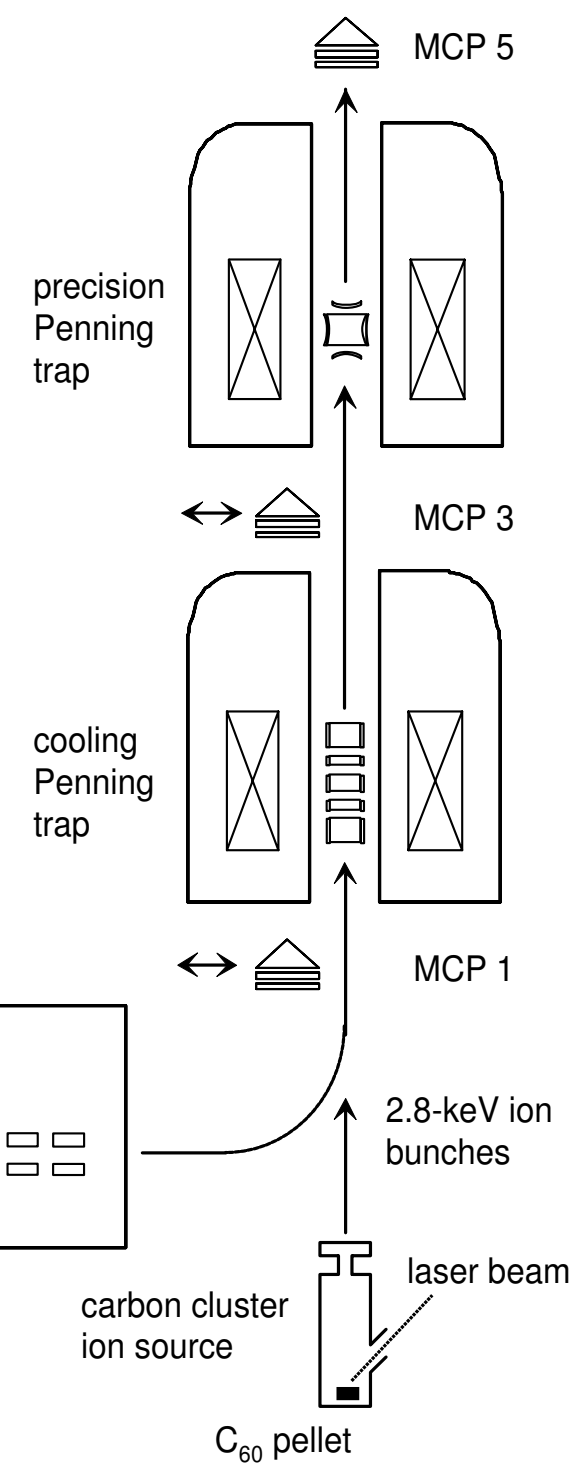

Figure 4: The triple-mass spectrometer ISOLTRAP installed at the on-line isotope separator ISOLDE/CERN. Micro-channel-plate (MCP) detectors are used to monitor the ion transfer as well as to record the time-of-flight resonance (MCP5) for the determination of the cyclotron frequency. The inset shows the cyclotron resonance curve of ${ }^{32} \mathrm{Ar}^{+}$after three hours measurement time with a fit of the theoretically expected line shape [53] to the data points.

The mass of ${ }^{34} \operatorname{Ar}\left(T_{1 / 2}=844 \mathrm{~ms}\right)$ was determined with a relative uncertainty of only $1.1 \cdot 10^{-8}$ [52]. This is the highest precision ever obtained in direct mass measurements of short-lived nuclides. With this result the $Q_{E C}$ value for the $\beta$ decay of ${ }^{34} \mathrm{Ar}$ was determined with an uncertainty well below $1 \mathrm{keV}$, sufficient to be used in a decisive test of Coulomb correction calculations for $f t$-values. In the very same context, also the $Q$ value of ${ }^{74} \mathrm{Rb}$ is important. Here ISOLTRAP was able to measure the mass of ${ }^{74} \mathrm{Kr}$ with a precision of $3.2 \cdot 10^{-8}$, but due to limited statistics the mass of ${ }^{74} \mathrm{Rb}$ could only be obtained with an uncertainty of $2.6 \cdot 10^{-7}$ or $\delta m=18 \mathrm{keV}$.

Resolution of nuclides in their ground and isomeric state has been demonstrated several times. For example, the low-lying levels of the $I=13 / 2$ neutron-deficient $\mathrm{Hg}$ isomers in the mid-neutron shell was resolved by ISOLTRAP mass spectrometry [50]. This measurement confirmed the coexistence of nuclear shapes at nearly degenerate 
energies $(\approx 100 \mathrm{keV})$ as previously deduced from laser and nuclear spectroscopy. Furthermore, the excitation energies of the isomeric states in the nuclei ${ }^{187,191} \mathrm{Hg}$ were determined by ISOLTRAP mass measurements for the first time [50].

ISOLTRAP mass measurements in long isotopic and isotonic chains allowed the study of the fine structure of the mass surface and clarified discontinuities in order to extract nuclear structure information from binding energies [50,57]. As an example for such a systematic survey, Fig. 5 shows two-neutron separation energies in the vicinity of $Z=82$ excluding (top) and including (bottom) ISOLTRAP data in the atomic mass evaluation.

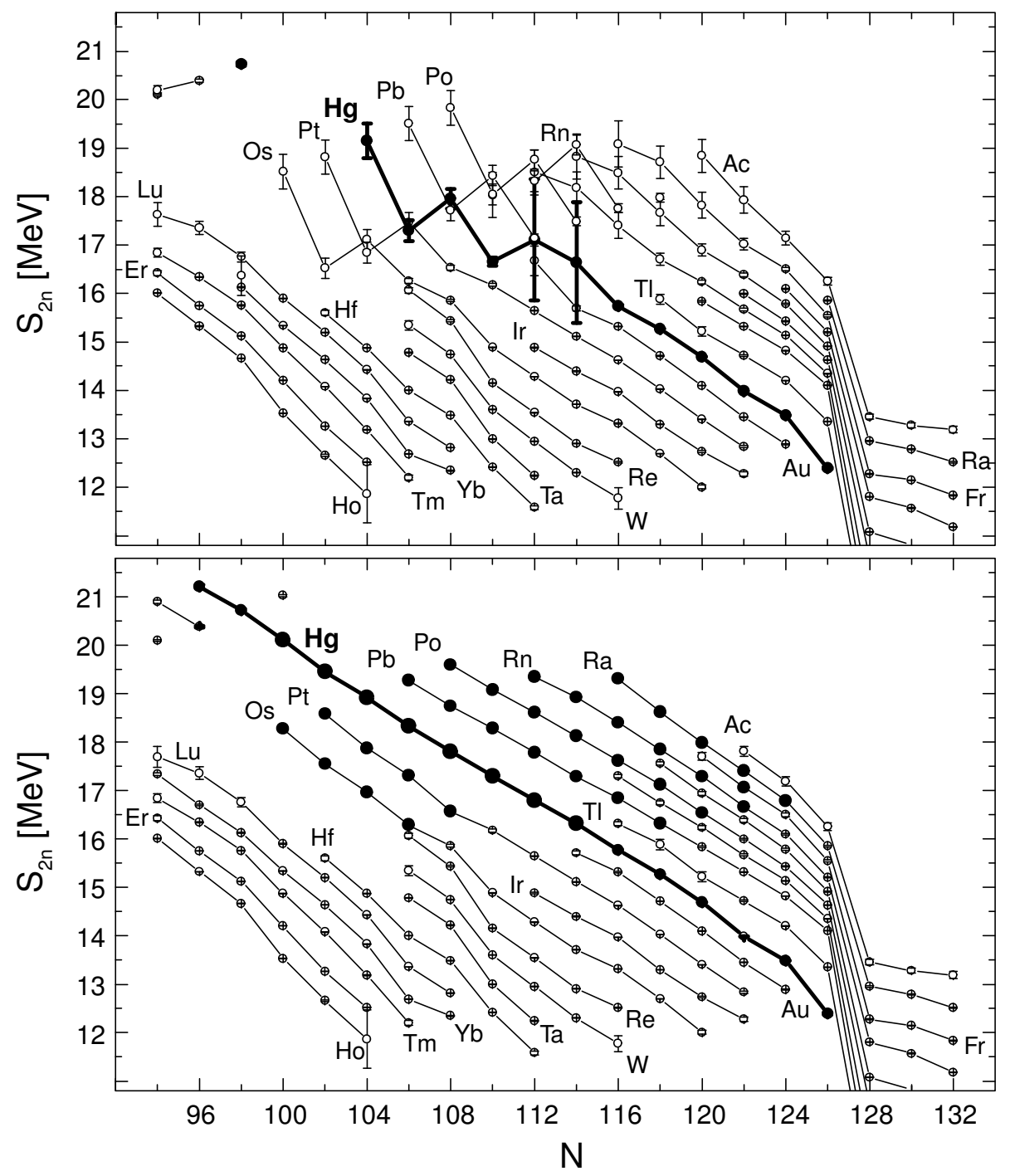

Figure 5: Two-neutron separation energies in the vicinity of $Z=82$ as a function of neutron number. Shown are $S_{2 n}$ values excluding (top) and including (bottom) ISOLTRAP data in the atomic mass evaluation.

\subsection{Planned Penning Trap Mass Spectrometer for ISOL beams}

At the ion guide ISOL facility (IGISOL) in Jyväskylä, a radio-frequency quadrupole buncher and cooler has recently been installed and tested very successfully [6]. A tremendous reduction in background as well as in line width was obtained for collinear laser spectroscopy by cooling and bunching the radioactive beam delivered 
by IGISOL [58,59]. This linear RFQ trap will also serve as injector for the Penning trap spectrometer JYFL-TRAP [24], which is tested right now. Here, mass measurements and decay studies on isotopes of refractory elements will be performed which are not available at ISOL facilities such as ISOLDE/CERN.

An ambitious project is TITAN [31] as planned at the ISAC on-line isotope separator at TRIUMF/Vancouver. Here, a set-up similar to the ISOLTRAP mass spectrometer is designed. However, an electron beam ion trap (EBIT) is placed between the RF quadrupole trap and the Penning trap, as shown in Fig. 6, which is used for charge breeding. In this way, higher cyclotron frequencies are obtained resulting in higher resolving power and accuracy, or vice versa, allow for a high-precision mass measurement in a much shorter time as compared to the case of singly-charged ions. This gives access to very short-lived isotopes. For example, tin isotopes are ionized by the strong electron beam of a powerful electron beam ion trap such as the Intense EBIT [60], which was designed and partly constructed at Livermore, to the Ne-like charge state $q=40+$ in $3 \mathrm{~ms}$. In addition, the TITAN facility offers quite a number of interesting options (Fig. 6) as, for example, efficient post-acceleration of radioactive ion beams.

Plans also exist for a trap facility at the Munich Accelerator for Fission Fragments (MAFF) to be installed at the new research reactor FRM II [26]. Here, especially the neutron-rich fission fragments produced from a uranium target by thermal neutrons will be investigated and used for fusion reactions after post-acceleration.

\subsection{REX-ISOLDE: Traps for Efficient Post-Acceleration}

REX-ISOLDE is a facility for post-acceleration of radioactive ion beams [27] which went into operation in 2001. The scheme is similar to that shown in Fig. 6 but instead of a RFQ a Penning trap (REX-TRAP) is used for accumulating, cooling, and bunching the ions delivered by ISOLDE. Furthermore, the structure of an electron beam ion source (EBIS) is used for charge-breeding which is longer than that of an $\mathrm{EBIT}$, and instead of mass measurements post-acceleration to $2.2 \mathrm{MeV} / \mathrm{u}$ is here the central issue. In order to increase the space charge limit ( $10^{7}$ ions) of REX-TRAP, which is the largest accumulator device based on a Penning trap [61], the rotatingwall techniques is foreseen [62].

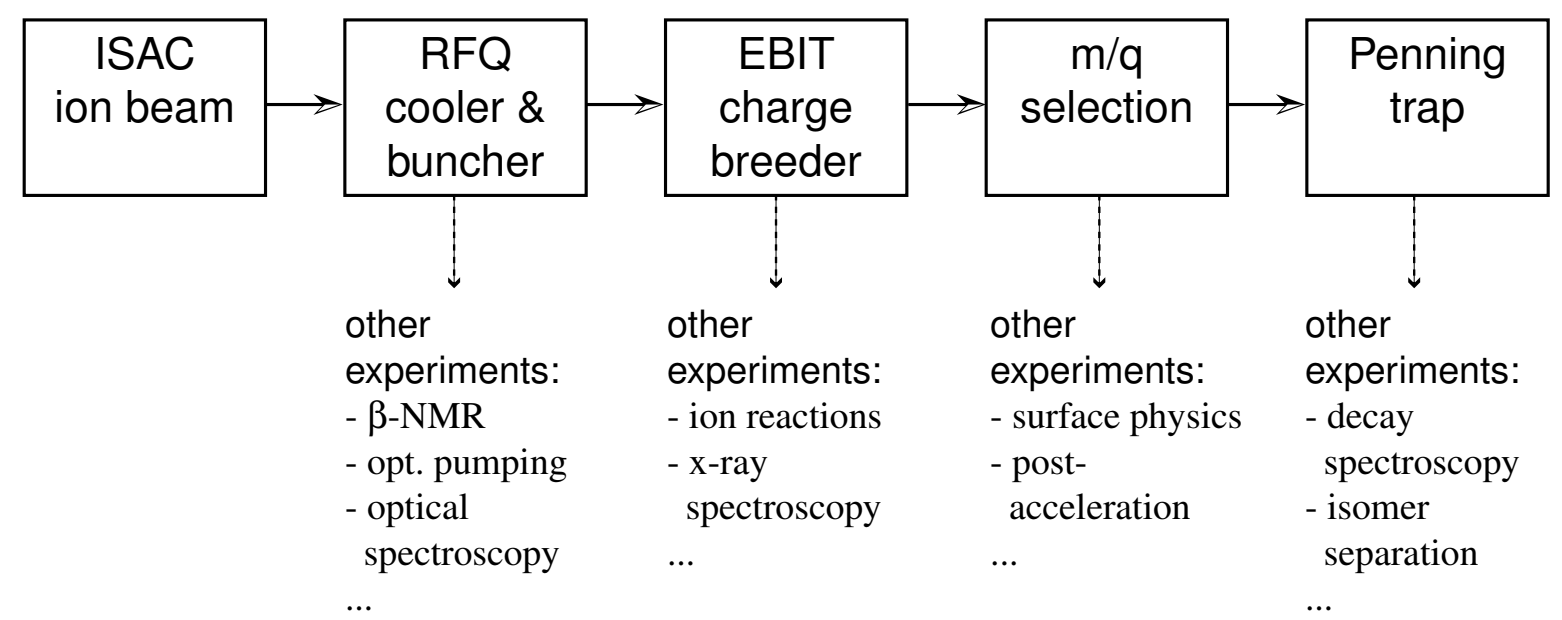

Figure 6: Schematics of the TITAN trap facility planned at the ISAC on-line isotope separator at TRIUMF, Vancouver. 


\subsection{Penning Trap Mass Spectrometers for MeV radioactive beams}

The second Penning trap for mass spectrometry of radionuclides which is operational is the Canadian Penning Trap (CPT) first developed for Chalk River and then transferred to Argonne [22,63]. CPT is quite similar to ISOLTRAP but uses a different production scheme for the nuclei under investigation and pioneered the gas cell technique for stopping fast reaction products. Here, the projectiles from the ATLAS linear accelerator hit a target where secondary fast beams are produced by fusion reactions. After separation in a Wien filter and in an Enge magnet, the ion beam is stopped in a cell filled with helium gas at a pressure of about 100 mbar. After stopping, the ions are guided to a nozzle by the gas flow and RF applied to disks forming a cone. Subsequently, they enter RFQ traps which are linear gas-filled and segmented structures. From here the ions are transferred as a bunch over several meters to another gas-filled RFQ structure mounted just below the precision Penning trap. This RFQ trap can be used for laser spectroscopy. Its main purpose, however, is cooling as well as bunched transfer of the ions to the Penning trap for mass determination.

A similar facility is the SHIPTRAP set-up [29] installed behind SHIP [64] in a GSIMunich Collaboration and tested presently. Here, fusion-evaporation products are separated from the primary beam by a Wien filter, stopped in a gas cell and transferred via linear RFQ structures to a Penning trap. Especially the investigation of very heavy radionuclides above uranium in the chart of nuclei is the scientific goal for this installation. Mass spectrometry, the search for optical transitions by laser spectroscopy above einsteinium [65], decay spectroscopy, and ion chemistry are planned.

At the National Superconducting Cyclotron Laboratory at Michigan State University, the LEBIT trap facility is presently under construction [25]. The goal of LEBIT is to demonstrate that secondary beams produced by projectile fragmentation at energies up to $200 \mathrm{MeV} / \mathrm{u}$ can be efficiently converted into low-energy beams and stored in ion traps. The scheme is similar to CPT and SHIPTRAP but new concepts are followed. These are cryogenic $(T \approx 80 \mathrm{~K})$ operation of the linear RFQ trap [25] and, first of all, the development of a He gas cell which is a completely new endeavor since it has to stop the very energetic beam from the fragment separator and must, therefore, be operated at a pressure as high as about 1 bar. LEBIT presents an important test case for the Rare Isotope Accelerator (RIA) project in the US [66] where a gas cell is a central device for post-acceleration of secondary radioactive ion beams.

\subsection{Penning Trap Mass Spectrometers for Highly-Charged lons}

As already discussed in Section 4.3 for the case of the TITAN facility, the use of highly-charged ions is advantageous for mass spectrometry in respect to resolving power and accuracy. At the Manne-Siegbahn Laboratory in Stockholm, the electron beam ion source CRYSIS is used for injection of stable highly-charged ions into the storage ring CRYRING and into the Penning trap spectrometer SMILETRAP [30].

Mass measurements could be performed up to mass $A=204$ with an accuracy of about $\delta \mathrm{m} / \mathrm{m}=2 \cdot 10^{-9}$ and close to $1 \cdot 10^{-10}$ in some cases where the ion under investigation and the reference ion for magnetic-field calibration were $\mathrm{g} / \mathrm{m}$-doublets. Important results are, for example: (i), the mass measurement of ${ }^{28} \mathrm{Si}$ required for a new definition of the kilogram which is the only SI-unit not related to atomic properties or fundamental constants [67]. A mass uncertainty of $0.35 \mathrm{ppb}$ was the final 
SMILETRAP result in agreement with the four times more accurate value obtained by the MIT group also by a Penning trap [68]; (ii), the determination of the mass difference of ${ }^{76} \mathrm{Ge}$ and ${ }^{76} \mathrm{Se}$ which represents an important constraint for the search for neutrino-less double beta decay [69]; (iii), the mass measurements of ${ }^{198} \mathrm{Hg}$ and ${ }^{204} \mathrm{Hg}$ [70] which are important for resolving the 5 to $12 \sigma$ discrepancy between the adjusted values in the Mass Tables by Audi and Wapstra [71] and the mass measurements of the Manitoba group by classical doublet mass spectrometry [72]. Since the SMILETRAP and the Manitoba results are in perfect agreement with each other, the reason for the discrepancy must be searched for in other input values of the Mass Tables.

The study of the nuclear half-life of ${ }^{77} \mathrm{Kr}$ as function of charge state $q$ is planned at LBL with the Penning trap RETRAP transferred from Livermore to Berkeley [26] ${ }^{77} \mathrm{Kr}$ will be produced by the cyclotron and then charge-breed in an EBIT to the nucleus with no or few electrons bound to it. This will allow one to investigate the bound-beta decay.

Mass measurements are also foreseen at the HITRAP facility planned at GSI [23]. The cyclotron frequency of the ${ }^{12} \mathrm{C}^{5+}$ ion in the GSI-Mainz experiment on the g-factor of the bound electron (see Section 5.4) could be measured with a resolving power of $10^{9}$, indicating a possible mass accuracy for highly-charged ions in HITRAP of the order of $\delta \mathrm{m} / \mathrm{m}=10^{-10}$ in the range of light ions up to fully stripped uranium or an uranium nucleus with one or few electrons attached to it.

\section{Studies of Fundamental Interactions and Symmetries}

By use of ion trap techniques it will become possible to observe nuclear decays in a very clean way and to study symmetries and properties of fundamental interactions as well as to test the Standard Model more precisely than it has been possible before.

\subsection{Decay Studies in Ion Traps}

Ion clouds stored in ion traps are very well suited for clean decay studies since there is no scattering from backing material. In the case of confinement in Penning traps, the $\gamma$-radiation can be detected undisturbed from $\beta$-particles by an annular detector in the bore of the superconducting magnet of the Penning trap. The $\beta$-particles or recoil ions, however, are guided efficiently by the magnetic field to the detectors placed outside the magnet $[17,36]$. In the case of Paul traps meshes can be used as electrode material [16] which allow for nearly $4 \pi$ solid angle for detection.

\subsection{Test of the Standard Model}

The study of super-allowed $\beta$-decays enables one to determine the weak vector coupling constant $\mathrm{g}_{\mathrm{v}}$ and in this way to test the unitarity of the Cabibbo-KobayashiMaskawa (CKM) matrix. Here, super-allowed $0^{+} \rightarrow 0^{+} \beta$ - and neutron-decay data indicate that the unitarity condition of the first row in the CKM-matrix is not fulfilled $[73,74]$. Besides precise branching ratios and half-lives very accurate Q-values are required. Some measurements have been performed recently at ISOLTRAP (see Section 4.3.1) and will be the goal of future experiments at the existing or planned Penning traps installed at accelerators. 
Precise measurements of the angular correlations in $\beta$-decay allow one to search for scalar or tensor contributions. Presently, the experiment on the $\beta$-delayed proton emission in ${ }^{32} \mathrm{Ar}$ sets the best limit [54]. Again, accurate masses are required. If not available, the isobaric multiplet mass equation (IMME) is used. The recent ${ }^{33} \mathrm{Ar}$ mass measurement [49] and follow-up experiments [55] showed that further tests of the IMME are required and direct mass measurements are essential as in the case of ${ }^{32} \mathrm{Ar}$. For the time being, it is an open question if the nearly $3 \sigma$-discrepancy between the IMME prediction for the mass of ${ }^{32} \mathrm{Ar}$ as used by Adelberger et al. [54] and the ISOLTRAP result [56] have a significant influence on the upper limit of a scalar contribution to weak interaction.

New experiments for angular correlation studies by use of ion traps are planned at GANIL [16] and Argonne [17]. A rather complex apparatus is presently being set up at ISOLDE: The WITCH experiment of the Leuven group [18] aims at a measurement of the energy spectrum of the recoiling ion after $\beta$-decay. Here, the nucleus ${ }^{35} \mathrm{Ar}$ is the first candidate for investigation. After trapping and cooling in a Penning trap the energy of the recoil ion is measured by an electrostatic retardation spectrometer similar to the one applied to determine an upper limit of the mass of the electron antineutrino [75]. Contributions of scalar interaction show up as a change of the energy spectrum of the recoil ion.

\subsection{Test of CPT Invariance}

The most precise test of CPT invariance in leptonic systems was obtained by a gfactor comparison of the electron and positron in a Penning trap experiment [76]. Again by use of a Penning trap mass spectrometer, a mass comparison of the proton and antiproton at the Low Energy Antiproton Ring LEAR at CERN yielded the most precise test of CPT in the baryonic sector [41]. Presently, two groups, the ATHENA [20] and ATRAP [21] Collaborations, pursue a test of the CPT invariance at the Antiproton Decelerator (AD) at CERN by a comparison of the $1 \mathrm{~s}-2 \mathrm{~s}$ transition frequency of hydrogen and antihydrogen. The accumulation of antiprotons and positrons in Penning traps works now routinely but the formation of antihydrogen by recombination has still to be demonstrated unquestionably. When that is achieved, the next challenge is to produce enough antihydrogen atoms and to cool and store those atoms in a neutral-atom trap for a long observation time so that two-photon laser spectroscopy of the $1 \mathrm{~s}-2 \mathrm{~s}$ transition can be applied.

\subsection{Test of Quantum Electrodynamics in Strong Fields}

Highly-charged ions provide a unique test ground for quantum electrodynamics (QED) in very strong electric and magnetic fields which is not accessible experimentally otherwise [77]. In recent years, very precise measurements and calculations have been performed on the Lamb shift in hydrogen-like and lithium-like heavy systems and also on the hyperfine structure splitting [78].

Another approach to test QED is the determination of the g-factor of the electron. The g-factor of the free electron has been measured to 12 digits by Dehmelt et al. [76] and represents the most stringent test of QED calculations [79] for weak electromagnetic fields. In the meantime, the CODATA Group has used this 
experimental value and the calculated one, taking QED as correct theoretical approach, to calculate the value of the fine structure constant [80].

In the case of a single electron bound to a nucleus, the g-factor is changed from the Dirac value $g_{J}=2$ to

$$
g_{J}=2 / 3\left(1+2 \sqrt{1-(Z \alpha)^{2}}\right)+C_{Q E D}+C_{f s}+C_{\text {recoil }}
$$

by relativistic effects, bound-state QED corrections, and still smaller corrections for finite nuclear size and recoil. In contrast to the situation of free particles and very light hydrogen-like systems, a perturbative treatment of the QED corrections in terms of $Z \alpha$ is not appropriate any longer. Instead, the QED contribution must be calculated in a non-perturbative manner to all orders of $Z \alpha$. A review can be found in [81] and further recent calculations in [82-84].

In close collaboration between GSI and the University of Mainz, an experimental setup with a new double-trap technique was developed in order to investigate the gfactor of the bound electron in hydrogen-like systems [85-88]. Here, the g-factor

$$
g_{J}=2 \frac{\omega_{L}}{\omega_{c}^{e}}=2 \frac{\omega_{L}}{\omega_{c}^{i}} \frac{\omega_{c}^{i}}{\omega_{c}^{e}}
$$

is measured as the ratio of the Larmor frequency $\omega_{L}$ of the electron spin to the cyclotron frequency of the electron $\omega_{c}^{e}$. The cyclotron frequency of the electron can be replaced by the measurable cyclotron frequency of the ion $\omega_{c}^{i}$ and the frequency ratio $\omega_{c}^{i} / \omega_{c}^{e}=m_{e} / m_{i}$ which is given by the well-known mass ratio of the electron and ion. The Larmor frequency is measured by observing the rate of spin flips of the electron as a function of the frequency $\omega_{m w}$ of the applied microwave field.

Figure 7 shows the central part of the experiment, the two cylindrical Penning traps which are kept at liquid-helium temperature [86]. A single hydrogen-like ion is created in the lower trap, called precision trap, by ionization with an electron beam, trapped, and cooled to $4 \mathrm{~K}$ by resistive cooling. This trap is located in the very homogeneous field of the superconducting magnet. Here, the cyclotron frequency $\omega_{c}^{i}$ is determined via a measurement of the eigenfrequencies (1-3) by the image current technique. In this precision trap also the microwave $\omega_{m w}$ is applied which induces eventually a change of the polarization of the bound electron. The spin direction is probed after transfer into the upper trap, called analysis trap. A ring electrode made of nickel adds a quadratic magnetic field component in z-direction which makes the axial oscillation frequency of the ion sensitive by $0.7 \mathrm{~Hz}$ to the electron spin polarization (see left part of Fig. 8). When the spin direction is determined, the ion is transferred back to the precision trap and a new try is performed by applying again the microwave field, transferring the ion to the analysis trap and by looking if a spin flip occurred.

The spin-flip rate for a specific $\omega_{m w}$ is determined in this way. Observing the spin-flip rate as a function of the frequency of the microwave one obtains a resonance curve as shown in the right part of Fig. 8. The center of the resonance yields the Larmor frequency. 
magnetic field lines

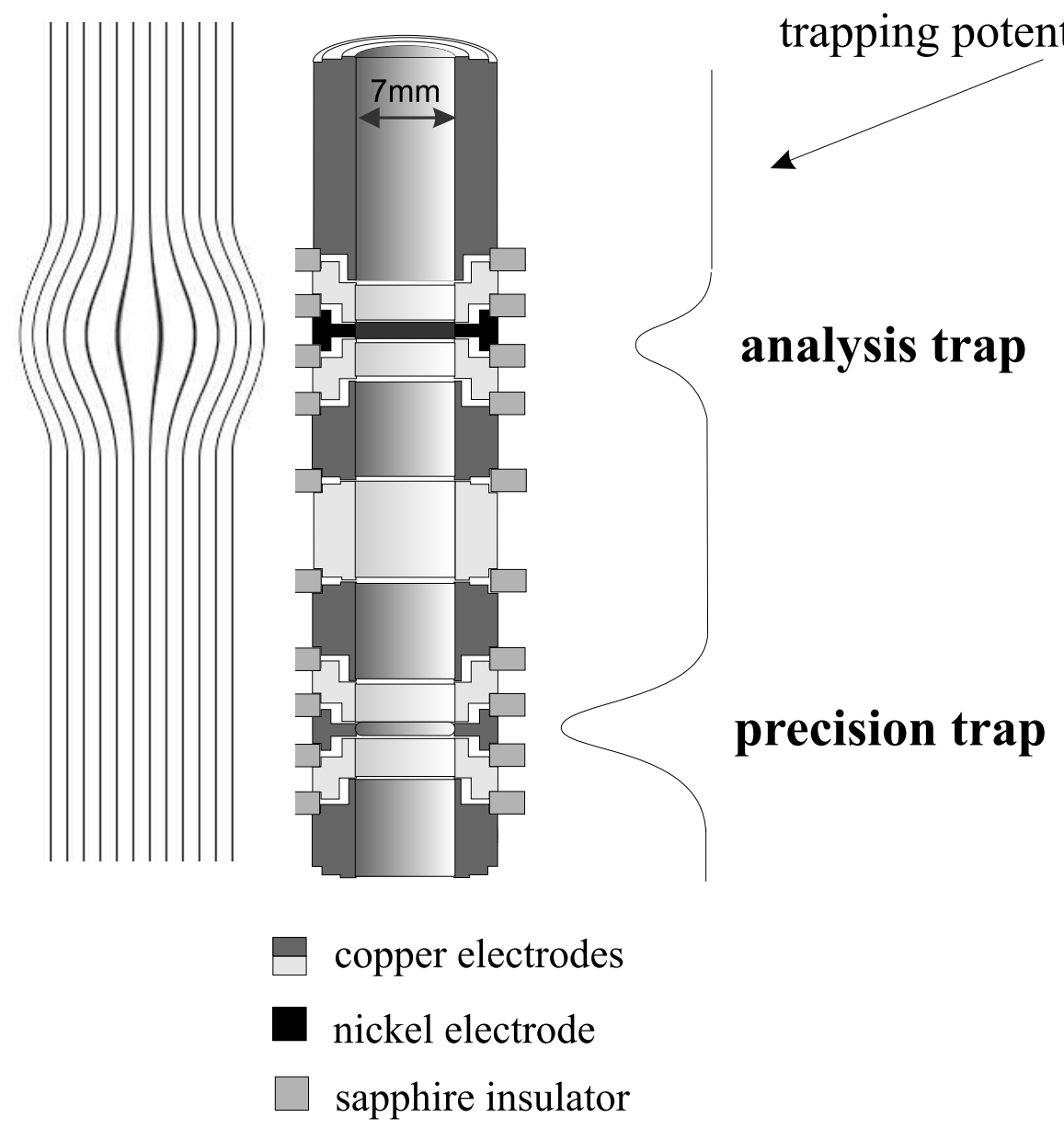

Figure 7: Penning trap arrangement for the determination of the g-factor of the bound electron in hydrogen-like systems. Two traps are used: In the homogeneous magnetic field of the lower trap spin-flip transitions are induced and the magnetic field is determined by a measurement of the cyclotron frequency. The upper trap and the inhomogeneous magnetic field serve for detection of the spin direction.
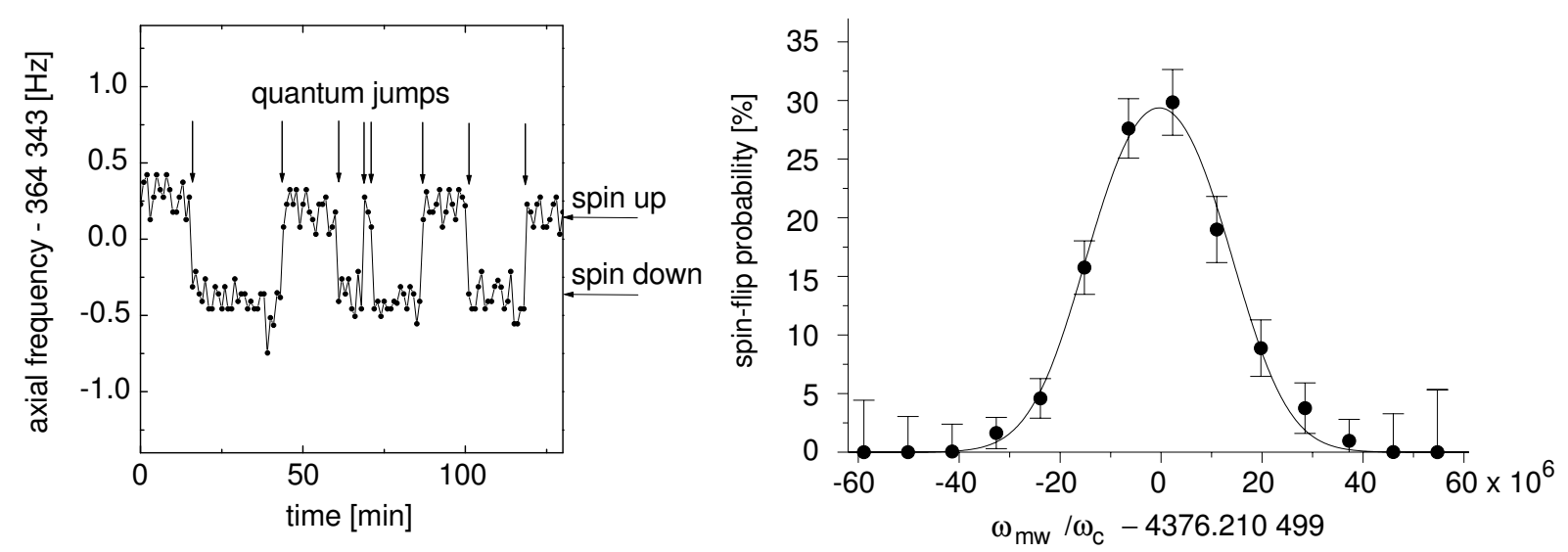

Figure 8: Detection of spin-flips (left) and spin-flip rate as a function of the frequency of the applied radio-frequency field (right). 
Experiments were performed on the hydrogen-like ions ${ }^{12} \mathrm{C}^{5+}[87]$ and ${ }^{16} \mathrm{O}^{7+}[88]$ with a total relative accuracy of $\delta g_{j} / g_{j}=2 \cdot 10^{-9}$. Here, the statistical error is about four times smaller than the error of the mass of the electron which enters (9). The experimental $g_{j}$-values agree within the total error bars with the theoretical ones, which take into account all orders in $(Z \alpha)$ for the bound-state QED contributions.

Recent theoretical work showed that the g-factor can be calculated with a still smaller relative uncertainty of only 1.5 to $3 \cdot 10^{-10}[83,84]$. Since the results for two hydrogenlike ions with different charge number are in very good agreement with the nonperturbative QED prediction, but the experimental error is dominated by the uncertainty of the electron mass, the argumentation can be turned around: Just as in the case of the g-factor of the free electron which defines now the fine structure constant, the QED predictions and the experimental values can be used to calculate the mass of the electron. This results in a decrease of the uncertainty of the electron mass by a factor of three [89] and, using more recent QED calculations, by a factor of four [90].

An ultimate test of bound-state QED will become possible if the HITRAP project [23] is realized (Fig. 9). Here, hydrogen-like ions can be produced by stripping the relativistic beams delivered by the GSI accelerator complex. These ions are injected into the storage ring ESR, cooled by electron cooling, decelerated to some $\mathrm{MeV} / \mathrm{u}$, and then injected into a linear decelerator. After deceleration to about $100 \mathrm{keV/q}$ the ions are accumulated and cooled in a cooler Penning trap before they are transferred to the set-up for g-factor measurements. As indicated in Fig. 9, such a facility will allow for, beside mass and g-factor measurements discussed above, quite a number of fascinating new investigations of highly-charged ions and their interaction with other particles and matter. In addition, a new determination of the fine structure constant is offered: When the correctness of the QED, finite size, and recoil corrections is demonstrated by a g-factor measurement of very heavy hydrogen-like systems, the dominating first term in Eq. (8) can be used to extract a new value of the fine structure constant from the measured g-factor in medium-heavy ions [91].

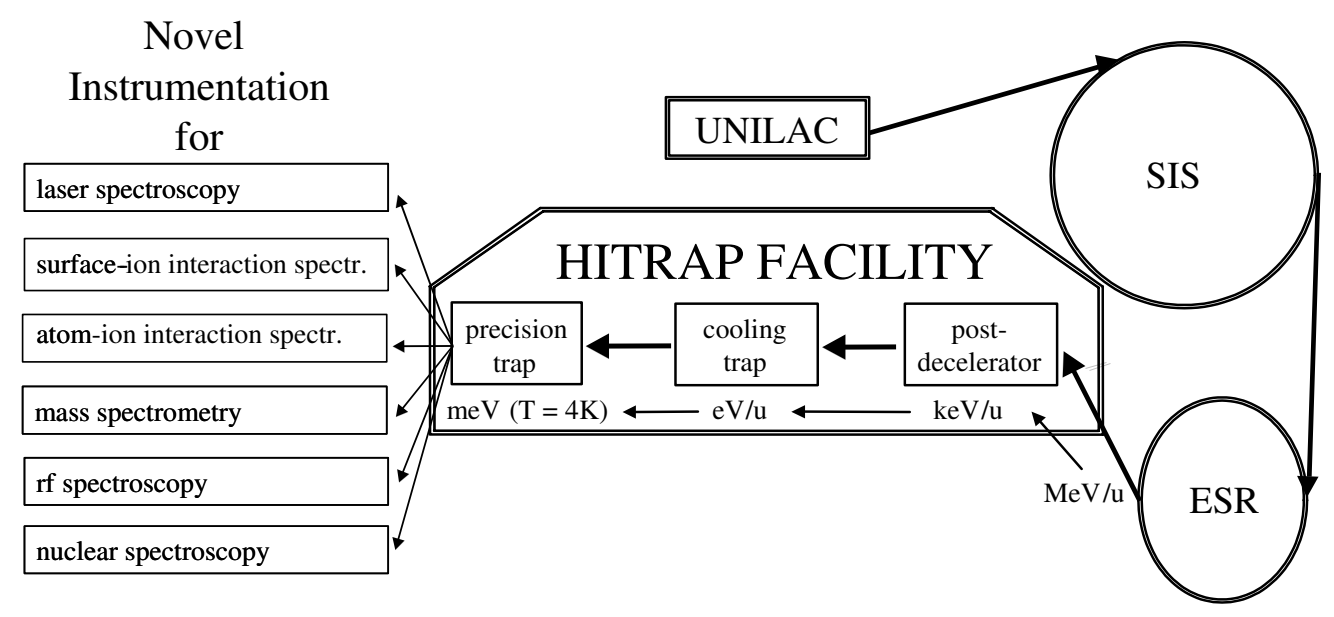

Figure 9: Schematics of the HITRAP project planned at GSI for experiments with highlycharged ions such as $\mathrm{U}^{91+}$ at nearly rest in space. 


\section{Conclusion}

Trapping and cooling of charged particles in ion traps have now become a versatile tool at accelerators which provides extreme accuracy and sensitivity as well as new approaches for atomic and nuclear physics experiments. Mass spectrometry of radionuclides in Penning traps is performed or planned at many radioactive beam facilities. Ion storage devices are or will be applied in the near future to high-precision proofs of quantum electrodynamics in strong fields, of the weak interaction, or to test symmetries and CPT invariance. They already play a central role at ISOL facilities as devices for beam manipulation and their application is planned in all future radioactive beam facilities.

Essential for the success of ion trap techniques was and is the support by the European Community. The SMILE-TRAP was developed within the SCIENCE program. Within the TMR program, the research network EUROTRAPS [92] and the RTD network EXOTRAPS [93] were supported. These networks, which finished end of 2001, were very fruitful, brought experimentalists and theoreticians together, stimulated the exchange of new ideas, and resulted, for example, in the development of the linear segmented RFQ trap and the g-factor measurements in hydrogen-like ions. The presently on-going RTD networks NIPNET [94], IONCATCHER [95], and HITRAP [96] promise a further evolution in trapping technologies and trap applications for atomic and nuclear physics.

\section{References:}

[1] G. Bollen et al., Nucl. Instrum. Methods A 368, 675 (1996).

[2] F. Herfurth et al., Nucl. Instrum. Methods A 469, 254 (2001).

[3] E. Kugler, Hyp. Int. 129, 23 (2000).

[4] A. Kellerbauer et al., Eur. Phys. J. D (2002), submitted.

[5] G. Savard et al., Eur. Phys. J. A (2002), in press.

[6] A. Nieminen et al., Nucl. Instrum. Methods A 469, 244 (2001).

[7] A. Kellerbauer et al., Nucl. Instrum. Methods A 469, 276 (2001).

[8] Proc. "Trapped Charged Particles and Fundamental Physics", Asilomar, CA, USA, 1998 (eds. D.H. Dubin and D. Schneider), AIP Conf. Proceedings 457 (1999)

[9] Proc. "Atomic Physics at Accelerators: Mass Spectrometry (APAC 2000), Cargése 2002, (eds. D. Lunney, G. Audi, H.-J. Kluge), Hyp. Int. 132 (2001)

[10] Proc. "14th International Conference on Electromagnetic Isotope Separation and Techniques Related to their Application (EMIS-14)" Conf., Victoria 2002, to be published in Nucl. Instrum. Methods B.

[11] Proc. "Trapped Charged Particles and Fundamental Interactions (TCPFI 2002)" Conf., Wildbad Kreuth 2002, to be published in J. Phys. B.

[12] R. S. Brown and G. Gabrielse, Rev. Mod. Phys. 58, 233 (1986).

[13] G. Bollen et al., J. Appl. Phys. 68, 4355 (1990).

[14] G. Savard et al., Phys. Rev. Lett. A 158, 247 (1991).

[15] L. Schweikhard et al., Int. J. Mass Spectrom. Ion Proc. 120, 71 (1992).

[16] E. Liénard et al., in: Proc. "Intern. Conf. On Nuclear Physics at the Border Line“, World Scientific, Singapore, 2001.

[17] G. Savard et al., Nucl. Phys. A 654, 961c (1999)

[18] D. Beck et al., in: Proc. TCPFI 1998 Conf., AIP Conf. Proc. 457, 172 (1999).

[19] D. Schneider, LLNL, private communication 
[20] L. V. Jørgensen et al. in "Plasma Physics IV" (eds. F. Anderegg, L. Schweikhard, C. F Driscoll), American Institute of Physics, New York, AIP Conf. Proc. 606, 35 (2002).

[21] G. Gabrielse et al., Phys. Lett. B 507,1 (2001); J. Walz", Proc. "Towards Laser Spectroscopy of Antihydrogen", (eds. H. Figger et al.) in "Laser Physics at the Limits", Springer, Berlin, Heidelberg, New York, p. 115 (2002)

[22] G. Savard et al., Hyp. Int. 132, 223 (2001).

[23] W. Quint et al., Hyp. Int. 132, 457 (2001).

[24] J. Szerypo et al., Acta Phys. Pol. B 32, 985 (2001).

[25] S. Schwarz et al., in: Proc. EMIS 2002 Conf., Nucl. Instrum. Methods B (2002), in press.

[26] J. Szerypo et al., in: Proc. EMIS 2002 Conf., Nucl. Instrum. Methods B (2002), in press.

[27] D. Habs et al., Hyp. Int. 129, 43 (2000).

[28] M. Wada et al., in: Proc. EMIS 2002 Conf., Nucl. Instrum. Methods B (2002), in press, and T. Nakamura et al., Opt. Comm. 205, 329 (2002).

[29] G. Sikler et al., in: Proc. EMIS 2002 Conf., Nucl. Instrum. Methods B (2002), in press and G. Marx et al., Hyp. Int. 132, 463 (2001).

[30] I. Bergström et al., Nucl. Instrum. Methods A 487, 618 (2002).

[31] J. Dilling et al., in: Proc. EMIS 2002 Conf., Nucl. Instrum. Methods B (2002), in press.

[32] G. Gabrielse et al., Phys. Rev. Lett. 63, 1360 (1989).

[33] P. H. Dawson, "Quadrupole Mass Spectrometry" (Elsevier, Amsterdam, 1976).

[34] A. Jokinen et al., in: Proc. EMIS 2002 Conf., Nucl. Instrum. Methods B (2002), in press

[35] J. Dilling, TRIUMF, Vancouver, Canada (private communication).

[36] L. Weissman et al., Hyp. Int. 132, 535 (2001).

[37] K. Blaum et al., in: Proc. EMIS 2002 Conf., Nucl. Instrum. Methods B (2002), in press.

[38] R. S. Van Dyck, Jr. et al., Phys. Rev. Lett. 19, 2888 (1993).

[39] E. A. Cornell et al., Phys. Rev. A 41, 312 (1990).

[40] G. Gräff et al., Z. Phys. A 297, 35 (1980).

[41] G. Gabrielse et al., Phys. Rev. Lett 82, 3198 (1999).

[42] D. L. Farnham, et al., Phys. Rev. Lett. 75, 3598 (1995).

[43] M. P. Bradley et al., Phys. Rev. Lett. 83, 4510 (1999).

[44] S. Rainville et al., in: Proc. TCPFI 2002 Conf., J. Phys. B (2002), to be published.

[45] K. Blaum et al., in: Proc. ENAM 2001 Conf., Eur. Phys. J. A (2002), in press.

[46] D. Beck et al., Nucl. Phys. A 626, 343c (1997).

[47] F. Ames et al., Nucl. Phys. A 651, 3 (1999).

[48] D. Beck et al., Eur. Phys. J. A 8, 307 (2000).

[49] F. Herfurth et al., Phys. Rev. Lett. 87, 142501 (2001).

[50] S. Schwarz et al., Nucl. Phys. A 693, 533 (2001).

[51] H. Raimbault-Hartmann et al., Nucl. Phys. A 706, 3 (2002).

[52] F. Herfurth et al., in: Proc. ENAM 2001 Conf., Eur. Phys. J. A (2002), in press.

[53] M. König et al., Int. J. Mass Spectrom. Ion Proc. 142, 95 (1995).

[54] E. G. Adelberger et al., Phys. Rev. Lett. 83, 1299 (1999); 83, 3101 (1999).

[55] M. C. Pyle et al., Phys. Rev. Lett. 88, 122501 (2002).

[56] K. Blaum et al., Phys. Rev. Lett. (2002), in preparation.

[57] G. Bollen, Nucl. Phys. A 693, 3 (2001).

[58] A. Nieminen et al., Phys. Rev. Lett. 88, 094801 (2002). 
[59] P. Campbell et al., Phys. Rev. Lett. 89, 082501 (2002)

[60] R. E. Marrs and D. R. Slaughter, UCRL-JC-131602.

[61] F. Ames et al., in: Proc. ENAM 1998 Conf., AIP Conf. Proc. 455, 927 (1998).

[62] F. Ames et al., in: Proc. EMIS 2002 Conf., Nucl. Instrum. Methods B (2002), in press.

[63] J. Clark et al., in: Proc. EMIS 2002 Conf., Nucl. Instrum. Methods B (2002), in press.

[64] S. Hofmann and G. Münzenberg, Rev. Mod. Phys. 72, 733 (2000).

[65] H. Backe et al., Phys. Rev. Lett. 80, 920 (1998).

[66] http://www.phy.anl.gov/ria/

[67] I. Bergström et al., Phys. Scripta 66, 1 (2002).

[68] F. Di Filippo et al., Phys. Rev. Lett. 73, 1481 (1994).

[69] G. Douysset et al., Phys. Rev. Lett. 86, 4259 (2001).

[70] T. Fritioff et al., Nucl. Phys. A (2002), in preparation.

[71] G. Audi and A. H. Wapstra, Nucl. Phys. A 595, 409 (1995).

[72] K. S. Kozier et al., Can. L. Phys. 58, 1311 (1980).

[73] J. C. Hardy and I. S. Towner, Hyp. Int. 132, 115 (2001).

[74] J. C. Hardy and I. S. Towner, in: Proc. ENAM 2001 Conf., Eur. Phys. J. A (2002), in press.

[75] J. Bonn et al., Prog. Part. Nucl. Phys. 48, 133 (2002).

[76] H. Dehmelt, Rev. Mod. Phys. 62, 525 (1990).

[77] P. J. Mohr, G. Plunien, and G. Soff, Phys. Rep. 293, 227 (1998).

[78] V. M. Shabaev et al., Phys. Scripta T 86, 7 (2000).

[79] V.W. Hughes and T. Kinoshita, Rev. Mod. Phys. 71, 133 (1999).

[80] P.J. Mohr and B.N. Taylor, Rev. Mod. Phys. 72, 351 (2000).

[81] T. Beier, Phys. Rep. 339, 79 (2000).

[82] T. Beier et al., Phys. Rev. A 62, 032510 (2000).

[83] V.M. Shabaev and V.A. Yerokhin, Phys. Rev. Lett. 88, 091801 (2002).

[84] V.A. Yerokhin et al., Phys. Rev. Lett. (2002), in press; see E-print archive, hepph/0205245 (2002), http://xxx.lanl.gov

[85] N. Hermanspahn et al., Phys. Rev. Lett. 84, 427 (2000).

[86] H. Häffner et al., Eur. Phys. J. D (2002), submitted.

[87] H. Häffner et al., Phys. Rev. Lett. 85, 5308 (2000).

[88] J. L. Verdú et al., Can. J. Ph. (in print)

[89] T. Beier et al., Phys. Rev. Lett. 88, 011603 (2002).

[90] T. Beier et al., Can. J. Ph. (in print)

[91] T. Beier et al., in: Proc. ENAM 2001 Conf., Eur. Phys. J. A15 (2002), in press.

[92] http://www.gsi.de/eurotraps

[93] http://www.phys.jyu.fi/research/igisol/exotraps/

[94] http://www.kvi.nl/ -trimp/web/html/nipnet.html

[95] http://www.ha.physik.uni-muenchen.de/

[96] http://www-linux.gsi.de/ hitrap/ 